Dedicated to the memory of the great scientist of Russian Physical Society- our colleague and friendLev Nikolaevich Lipatov (02.05.1940-04.09.2017)

\title{
Ternary $S U(3)$-group symmetry and its possible applications in hadron-quark substructure. Towards a new spinor-fermion structure
}

\author{
Alexander Maslikov ${ }^{1}$ and Guennady Volkov ${ }^{2}$ \\ ${ }^{1}$ Dubna University, "Protvino" Branch, Protvino, Russia \\ ${ }^{2}$ RSC KI Peterbourg Nuclear Physics Institute, Gatchina, St-Peterbourg, Russia
}

\begin{abstract}
The questions on the existence of the three color quark symmetry and three quark-lepton generations could have the origin associated with the new exotic symmetries outside the Cartan-Killing-Lie algebras / groups. Our long-term search for these symmetries has been began with our Calabi-Yau space classification on the basis of the $n$-ary algebra for the reflexive projective numbers and led us to the expansion of the binary $n=2$ complex and hyper complex numbers in the framework of the n-ary complex and hyper-complex numbers with $n=3,4, \ldots$ where we constructed new Abelian and non-Abelian symmetries. We have studied then norm-division properties of the Abelian nary complex numbers and have built the infinite chain of the Abelian groups $U^{(n-1)}=[U(1) \times \ldots \times U(1)]_{(n-1)}$. We have developed the n-ary holomorphic (polymorphic) analysis on the n-ary complex space $\mathbf{N} \mathbf{C}^{\{n\}}$, which led us to the generalization of the quadratic Laplace equations for the harmonic functions. The generalized Laplace equations for the $n$-ary harmonic functions give us the n-th order homogeneous differential equations which are invariant with respect to the Abelian n-ary groups $U^{(n-1)}$ and with some new spatial properties. Further consideration of the non-Abelian n-ary hyper-complex numbers opens the infinite series of the non-Abelian $T_{n} S U(n)$-Lie groups $(\mathrm{n}=3,4, \ldots)$ and its corresponding $t_{n} s u(n)$ algebras. One of the exceptional features of these symmetry groups is the appearance of some new n-dimensional spinors that could lead to an extension of the concept of the $S U(2)$-spin, to the appearance of n-dimensional quantum structures -exotic "n-spinor" matter $(n=3,4, \ldots$ - maarcrions). It is natural to assume that these new exotic "quantum spinor states" could be candidates for the pra-matter of the quark-charge leptons or/and for the dark matter. We will be also interested in the detection of the exotic quantum 'n-spinor" matter in the neutrino and hadron experiments.
\end{abstract}

Geometrical basis of modern quantum physics of quarks and leptons - Standard Model can be represented as a space-time $D=(3+1)$ - four-dimensional continuum. The symmetry properties of this continuum are based on the space-time Lorentz-Poincare symmetry group $P=S O(1,3) \ltimes R^{4}$ and the corresponding quantum theory is constructed on the basis of the groups of internal symmetries $S U\left(3^{C}\right) \times S U(2)_{I} \times U(1)_{Y}$. At present, one of the central theoretical questions for the Standard Model concerns its geometrization. By geometrization we mean the existence of definite connections between the space-time geometry with the laws of 
motion in it, described by the global and local external symmetries, and the fundamental components of the matter and radiation moving in this space-time geometry, with the quantum properties and the interactions, described by the local and global internal symmetries. Such quantum numbers for the quark-lepton matter as isotope-spin, color, the number of the quarklepton generations, described by the corresponding internal symmetries, could be understood within the framework of geometrization of the Standard Model. In our understanding, the geometrization of the SM could mean solving such an important problem as the relationship between the internal and external symmetries which could relate the internal quantum properties of the matter with geometric features of the ambient space-time. In our opinion, the questions of expanding the fundamentals of the quantum physics can arise on the way of the proton-neutron and/or the electron quantization, on searching for the geometric beginning of the 3-quark-color nature and the 3(3+1)-quark-lepton generations, unique properties of the 3 -neutrinos and the unusual quantum nature of dark matter.

In the search for the new geometric varieties and related groups (both external and internal) symmetries and their representations in the framework of extensions of the well-studied theory of the binary $n=2$ complex and hyper-complex numbers to the theory of the n-ary complex (Abelian) and hyper-complex (non-Abelian) numbers with $n>2$. The geometrical nature of the n-ary Abelian groups could be searched for in the theory of the Abelian $n$-ary complex numbers. We have taken the idea of considering the $n$ - ary complex numbers in the Euclidean $R^{n}$ - spaces as a tool to find new symmetries in connection with the Calabi-Yau space classification of any dimension $C Y(d), d=$ complex.dim. $=2 p$-real.dim., what we have done on the basis of the $n$ - ary theory of the reflexive projective numbers $[1,2]$. The $C Y(d)$-spaces $d=2,3, \ldots$ are the multi-dimensional generalizations of the $d=1$ one-dimensional torus. This classification allowed us to see new-n-ary structures in Newtonian polyhedrons for the Calabi-Yau spaces $C Y(d)$ with the holonomy group $S U(d)$, $d=3,4,5, \ldots[1,2]$ During the latest 20 year period we have related this development with the search for the new algebraic structures and symmetries based on the theories of the normdivision algebras for the n-ary complex and then to the hyper-complex numbers for $n>2$ : firstly to the Abelian cyclic $C_{n}$-[3-7] and, then to the non-Abelian extensions of them[8]. The most intriguing results of this method are related to more complicated algebraic structures of the corresponding norm-division algebras. According to the Abelian $C_{n}$-cyclic group the complexification of the Euclidean $\mathbb{R}^{n}$ spaces which followed by this method we have consequently constructed the series $-n=3,4,5,6,12$ of the $n$-dimensional $(n-1)$-parameter Abelian group-hypersurfaces with $n=2,3, \ldots$ We have determined the Abelian group symmetries for these spaces. The n-ary complex numbers have led to two isomorphic, n-ary "unitary" and "orthogonal", Abelian (n-1)-parametric symmetry groups, which could be the basis to describe the invisible light of the Universe [5, 10, 11]. The feature of our approach is the appearance of noncompact Abelian symmetry groups. The theory of Abelian complex numbers is based on the complexification of the Euclidean $\mathbb{R}^{n}$-space[4, 5]:

$$
z=x_{0} q_{0}+x_{1} q+\ldots+x_{(n-1)} q^{(n-1)}
$$

using $C_{n}=q_{0}, q, \ldots, q^{(n-1)}: q^{n}= \pm q_{0}, q_{0}-$ unit - cyclic groups of their n-one-dimensional irreducible representations for conjugation operations:

$$
\tilde{q}=q^{\{1\}}=j q, \tilde{\tilde{q}}=q^{\{2\}}=j^{2} q, \ldots, q^{\{n-1\}}=j^{(n-1)} q ; \quad q^{\{n\}}=q ; \quad j=e^{(2 \pi i / n)}
$$

which allow one to determine the norm $\|z\|^{n}=z \cdot z^{\{1\}} \cdot \ldots \cdot z^{\{n-1\}}$, which has composite group properties $\left\|z_{1} \cdot z_{2}\right\|^{n}=\left\|z_{1}\right\|^{n} \cdot\left\|z_{2}\right\|^{n}$, which allows for $\mathrm{n}$-ary complex numbers with a single norm to determine (n-1)-parametric Abelian groups. Following the Abelian $C_{n}$-complexification of Euclidean spaces $R^{n}$, we successively construct the series of Abelian (n-1)-parameterinvariant hypersurfaces $\|z\|^{n}=F_{0}\left(x_{0}, \ldots, x_{(n-1)}\right)=1$ for $n=3,4,5,6, \ldots, 12$ (further expansion 
is obvious) we study the process, we derive Euler formulae as the basis to derive n-aryunitary Abelian groups in the $(n \times n)$-matrix representation. For illustration we present the expressions of algebraic equations for the hypersurfaces defined by the $C^{n}$, - cyclic unit numbers only for $n \leq 6$ for both cases, A) $q^{n}=q_{0}$, B) $q^{n}=-q_{0}$, respectively, (these cases can be linked by extended Wick twist) [9]:

\begin{tabular}{||c|c||c||}
\hline \hline \hline$q_{n}^{n}$ & $\tilde{q}_{n}=e^{(2 \pi i / n)} q$ & $\left\langle z z^{1} \ldots z^{n-1}\right\rangle=R_{n}^{n}$ \\
\hline \hline \hline$i^{2}=-1$ & $\bar{i}=-i$ & $x_{0}^{2}+x_{1}^{2}=R^{2}$ \\
\hline \hline$q_{2}^{2}=1$ & $\tilde{q}_{2}=-q_{2}$ & $x_{0}^{2}-x_{1}^{2}=H^{2}$ \\
\hline \hline \hline$q_{3}^{3}=1$ & $\tilde{q}_{3}=e^{(2 \pi i / 3)} q_{3}$ & $x_{0}^{3}+x_{1}^{3}+x_{2}^{3}-3 x_{0} x_{1} x_{2}=R^{3}=\rho \cdot r^{2}$ \\
\hline \hline \hline$q_{4}^{4}=1$ & $\tilde{q}_{4}=e^{(\pi i / 2)} q_{4}$ & $\left.\left[\left(x_{0}-x_{2}\right)^{2}+\left(x_{1}-x_{3}\right)^{2}\right] \cdot\left[x_{0}+x_{2}\right)^{2}-\left(x_{1}+x_{3}\right)^{2}\right]=H_{1}^{2} \cdot H_{2}^{2}$ \\
\hline & & {$\left[x_{3}^{2}+x_{1}^{2}-2 x_{0} x_{2}\right]^{2}-\left[x_{0}^{2}+x_{2}^{2}-2 x_{1} x_{3}\right]^{2}=R_{1}^{4}-R_{2}^{4}$} \\
\hline \hline$q_{4}^{4}=-1$ & $\tilde{q}_{4}=e^{(\pi i / 2)} q_{4}$ & {$\left[x_{3}^{2}-x_{1}^{2}+2 x_{0} x_{2}\right]^{2}+\left[x_{0}^{2}-x_{2}^{2}+2 x_{1} x_{3}\right]^{2}=R_{1}^{4}+R_{2}^{4}=1$} \\
\hline \hline$q_{5}^{5}=1$ & $\tilde{q}_{5}=e^{(2 \pi i / 5)} q_{5}$ & $x_{0}^{5}+x_{1}^{5}+x_{2}^{5}+x_{3}^{5}+x_{4}^{5}-5 x_{0} x_{1} x_{2} x_{3} x_{4}-$ \\
& & $-5\left\{x_{0}^{3}\left(x_{1} x_{4}+x_{2} x_{3}\right)+x_{1}^{3}\left(x_{0} x_{2}+x_{3} x_{4}\right)+x_{2}^{3}\left(x_{1} x_{3}+x_{0} x_{4}\right)\right.$ \\
& & $\left.+x_{3}^{3}\left(x_{1} x_{3}+x_{0} x_{4}\right)+x_{4}^{5}\left(x_{0} x_{3}+x_{1} x_{2}\right)\right\}$ \\
& & $+5\left\{x_{0}\left(x_{1}^{2} x_{4}^{2}+x_{2}^{2} x_{3}^{2}\right)+x_{1}\left(x_{0}^{2} x_{2}^{2}+x_{3}^{2} x_{4}^{2}\right)\right.$ \\
& & $\left.+x_{2}\left(x_{1}^{2} x_{3}^{2}+x_{4}^{2} x_{0}^{2}\right)+x_{3}\left(x_{2}^{2} x_{4}^{2}+x_{0}^{2} x_{1}^{2}\right)+x_{4}\left(x_{3}^{2} x_{0}^{2}+x_{1}^{2} x_{2}^{2}\right\}\right)$ \\
\hline \hline
\end{tabular}

\begin{tabular}{|c|c|c|}
\hline$q_{6}^{6}=1$ & $\tilde{q}_{6}=e^{(\pi i / 3)} q_{6}$ & $\begin{array}{c}{\left[\left(x_{0}+x_{3}\right)^{3}+\left(x_{1}+x_{4}\right)^{3}+\left(x_{2}+x_{5}\right)^{3}-3\left(x_{0}+x_{3}\right)\left(x_{1}+x_{4}\right)\left(x_{2}+x_{5}\right)\right]} \\
\cdot\left[\left(x_{0}-x_{3}\right)^{3}+\left(x_{1}-x_{4}\right)^{3}+\left(x_{2}-x_{5}\right)^{3}-3\left(x_{0}-x_{3}\right)\left(x_{1}-x_{4}\right)\left(x_{2}-x_{5}\right)\right] \\
=\left[u_{0}^{3}+u_{1}^{3}+u_{2}^{3}-3 u_{0} u_{1} u_{2}\right] \cdot\left[v_{0}^{3}+v_{1}^{3}+v_{2}^{3}-3 v_{0} v_{1} v_{2}\right] \\
=\left\{F_{1}\right\}^{3} \cdot\left\{F_{2}\right\}^{3}=\rho_{1} r_{1}^{2} \cdot \rho_{2} r_{2}^{2}=1\end{array}$ \\
\hline & & $\begin{array}{c}\left\{\left[\left(x_{0}^{3}+x_{2}^{3}+x_{4}^{3}-3 x_{0} x_{2} x_{4}\right)\right]\right. \\
\left.-3\left[x_{0}\left(x_{3}^{2}-x_{1} x_{5}\right)+x_{2}\left(x_{5}^{2}-x_{1} x_{3}\right)+x_{4}\left(x_{1}^{2}-x_{3} x_{5}\right)\right]\right\}^{2}- \\
-\left\{\left[\left(x_{1}^{3}+x_{3}^{3}+x_{5}^{3}-3 x_{1} x_{3} x_{5}\right]\right.\right. \\
\left.+3\left[x_{1}\left(x_{4}^{2}-x_{0} x_{2}\right)+x_{3}\left(x_{0}^{2}-x_{2} x_{4}\right)+x_{5}\left(x_{2}^{2}-x_{0} x_{4}\right)\right]\right\}^{2} \\
=\left\{F_{1}^{3}\right\}^{3}-\left\{F_{2}^{3}\right\}^{2}=1\end{array}$ \\
\hline$q_{6}^{6}=-1$ & $\tilde{q}_{6}=e^{(\pi i / 3)} q_{6}$ & $\begin{array}{c}\left\{\left[\left(x_{0}^{3}+x_{2}^{3}-x_{4}^{3}+3 x_{0} x_{2} x_{4}\right)\right]\right. \\
\left.-3\left[x_{0}\left(x_{3}^{2}-x_{2} x_{4}\right)+x_{2}\left(x_{5}^{2}+x_{1} x_{3}\right)-x_{4}\left(x_{1}^{2}+x_{3} x_{5}\right)\right]\right\}^{2}+ \\
+\left\{\left[x_{1}^{3}-x_{3}^{3}+x_{5}^{3}+3 x_{1} x_{3} x_{5}\right]\right. \\
\left.\left.-3\left[x_{1}\left(x_{4}^{2}-x_{0} x_{2}\right)\right)-x_{3}\left(x_{0}^{2}+x_{2} x_{4}\right)+x_{5}\left(x_{2}^{2}+x_{0} x_{4}\right)\right]\right\}^{2} \\
=\left\{\tilde{F}_{1}^{3}\right\}^{2}+\left\{\tilde{F}_{2}^{3}\right\}^{2}=1\end{array}$ \\
\hline
\end{tabular}

Generalization of the n-dimensional trigonometry and the Pythagorean theorems for $\mathrm{n}$ dimensional simplexes in each n-ary case has been obtained, for example, let see $n=3$ :

$$
e^{\left(q \alpha+q^{2} \beta\right)}=c_{0}(\alpha, \beta) q_{0}+s_{0}(\alpha, \beta) q+t_{0}(\alpha, \beta) q^{2},
$$

where $c_{0}^{3}+s_{0}^{3}+t_{0}^{3}-3 c_{0} s_{0} t_{0}=1$. The two parameter Abelian ternary "unitarity" is:

$$
U=e^{\left(\alpha q+\beta q^{2}\right)}, U^{+}=e^{\left(j \alpha q+j^{2} \beta\right) q^{2}}, U^{++}=e^{\left.\left(j^{2} \alpha q+j \beta\right) q^{2}\right)}: \quad U \cdot U^{+} \cdot U^{++}=\hat{1},
$$

or in the matrix form:

$$
\begin{aligned}
& U=\left(\begin{array}{ccc}
a & q b & q^{2} c \\
c q^{2} & a & q b \\
q b & c q^{2} & a
\end{array}\right), U^{+}=\left(\begin{array}{ccc}
a & j q b & j^{2} q^{2} c \\
j^{2} c q^{2} & a & j q b \\
j q b & j^{2} c q^{2} & a
\end{array}\right), U^{++}=\left(\begin{array}{ccc}
a & j^{2} q b & j q^{2} c \\
j c q^{2} & a & j^{2} q b \\
j^{2} q b & j c q^{2} & a
\end{array}\right) \\
& U \cdot U^{+} \cdot U^{++}=\operatorname{det} U=\left(a^{3}+b^{3}+c^{3}-3 a b c\right) \cdot \hat{1}=\hat{1} .
\end{aligned}
$$


Subsequent consideration in $C N^{(n)}-$ n-ary complexified $\mathbf{R}^{n}$ - Euclidean space the ways of the holomorphizm (and polymorphizm) for the functions [3], [4]:

$$
F\left(z, z^{\{1\}}, \ldots, z^{\{n-1\}}\right)=F_{0}\left(x_{0}, \ldots, x_{(n-1)}\right) q_{0}+F_{1}\left(x_{0}, \ldots, x_{(n-1)}\right) q+\ldots+F_{(n-1)}\left(x_{0}, \ldots, x_{(n-1)} q^{\{n-1\}}\right.
$$

$\left(z^{\{p\}}-p=1,2, \ldots,(n-1)\right.$ is the number of the conjugation operations of the $n$-ary complex number $\mathrm{z}$ ) which allows us to derive n-dimensional wave equations (of the Laplace / Dirac type) for the harmonic functions $F_{a}\left(x_{0}, \ldots, x_{(n-1)}\right)$ and the corresponding n-spinors, invariant relativity of the corresponding $(n-1)$-parametric Abelian symmetry groups. The main conclusion of our calculations is that the Abelian properties of the cyclic groups $C_{n}$ lead to the factorization of all the corresponding group $(n-1)$-dimensional hypersurfaces $(n-1)$ with the definition of the Abelian group $U^{(n-1)}$, and by analyzing $U^{(n-1)}$ - the invariant Laplace differential equations for arity-n harmonic functions $F_{i}\left(x_{0}, \ldots, x_{(n-1)}\right), i=0, \ldots, n-1$, where the harmonic functions are determined through decomposition of the holomorphic function $[3,8]$.

We have identified and investigated all the Abelian symmetries for the cases $(n=$ $3, \ldots, 12$ ), which could serve as opportunities to consider Abelian theories of invisible light of the Universe, interacting with some invisible matter - exotic n-spinor "maarkrions". In this view, we have naturally extended the constructions of Abelian n-ary complex numbers studied to the construction of the non-Abelian n-ary hyper-complex numbers starting from two introducing hyper-complex numbers producing the $\left(n^{2}-1\right)$ (for $\left.t s u(3)-8\right)$ generators [8]:

$$
Z=x_{0} Q_{0}+x_{1} Q_{1}+\ldots+x_{\left(n^{2}-1\right)} Q_{\left(n^{2}-1\right)}:\left(Q_{k}\right)^{n}= \pm Q_{0} ; k=1, \ldots, n^{2}-1 .
$$

The Laplace differential equations can be found from the Cauchy-Riemann equations

$$
\frac{\partial F\left(z, z^{\{1\}}, \ldots, z^{\{n-1\}}\right)}{\partial z^{\{1\}}}=0 \ldots, \frac{\partial F\left(z, z^{\{1\}}, \ldots, z^{\{n-1\}}\right)}{\partial z^{\{n-1\}}}=0 \ldots
$$

of the order deg $=\mathrm{n}$. Following the Dirac procedure one can get from the differential equations for $n$ - ary harmonic functions the linear differential invariant equations for $n-$ spinors:

$$
\hat{F}\left(\partial x_{0}, \ldots, \partial x_{n-1}\right) \psi=0, \quad \psi=\left(\begin{array}{c}
\eta_{0} \\
\ldots \\
\eta_{n-1}
\end{array}\right)
$$

As an example we give the commutation relations for $t s u(3)$-algebra [8]:

$$
\begin{aligned}
& {\left[Q_{1}, Q_{2}\right]=\left(j^{2}-j\right) Q_{6} ;\left[Q_{2}, Q_{3}\right]=\left(j^{2}-j\right) Q_{4} ;\left[Q_{3}, Q_{1}\right]=\left(j^{2}-j\right) Q_{5}} \\
& {\left[Q_{4}, Q_{5}\right]=\left(j^{2}-j\right) Q_{3} ;\left[Q_{5}, Q_{6}\right]=\left(j^{2}-j\right) Q_{1} ;\left[Q_{6}, Q_{4}\right]=\left(j^{2}-j\right) Q_{2}} \\
& {\left[Q_{1}, Q_{4}\right]=0 ;\left[Q_{2}, Q_{4}\right]=\left(j^{2}-j\right) Q_{8} ;\left[Q_{3}, Q_{4}\right]=\left(j-j^{2} Q_{7}\right.} \\
& {\left[Q_{1}, Q_{5}\right]=\left(j-j^{2}\right) Q_{7} ;\left[Q_{2}, Q_{5}\right]=0 ;\left[Q_{3}, Q_{5}\right]=\left(j^{2}-j\right) Q_{8}} \\
& {\left[Q_{1}, Q_{6}\right]=\left(j^{2}-j\right) Q_{8} ;\left[Q_{2}, Q_{6}\right]=\left(j-j^{2}\right) Q_{7} ;\left[Q_{3}, Q_{6}\right]=0} \\
& {\left[Q_{1}, Q_{7}\right]=\left(j-j^{2}\right) Q_{3} ;\left[Q_{1}, Q_{8}\right]=\left(j^{2}-j\right) Q_{2} ;\left[Q_{2}, Q_{7}\right]=\left(j-j^{2}\right) Q_{1}} \\
& {\left[Q_{2}, Q_{8}\right]=\left(j^{2}-j\right) Q_{3} ;\left[Q_{3}, Q_{7}\right]=\left(j-j^{2}\right) Q_{2} ;\left[Q_{3}, Q_{8}\right]=\left(j^{2}-j\right) Q_{1}} \\
& {\left[Q_{4}, Q_{7}\right]=\left(j^{2}-j\right) Q_{5} ;\left[Q_{4}, Q_{8}\right]=\left(j-j^{2}\right) Q_{6} ;\left[Q_{5}, Q_{7}\right]=\left(j^{2}-j\right) Q_{6} ;} \\
& {\left[Q_{5}, Q_{8}\right]=\left(j-j^{2}\right) Q_{4} ;\left[Q_{6}, Q_{7}\right]=\left(j^{2}-j\right) Q_{4} ;\left[Q_{6}, Q_{8}\right]=\left(j-j^{2}\right) Q_{5}}
\end{aligned}
$$


The unit ternary-hypercomplex numbers produce the non-Abelian ternary-unitary STU(3)-group Lee. Applying the unusual comutation rules we have constructed the hypersurface defined by the unit ternary:

$$
z=\left(x_{0} q_{0}+x_{7} q+x_{8} q^{2}\right)+\left(x_{1} q_{0}+x_{2} q+x_{3} q^{2}\right) q_{1}+\left(x_{4} q_{0}+x_{5} q+x_{6} q^{2}\right) q_{1}^{2} .
$$

The 8 imaginary units $\left\{Q_{k}\left|q, q^{2}, q_{1}, q q_{1}, q^{2} q_{1}, q_{1}^{2}, q q_{1}^{2}, q_{1}^{2}, q^{2}\right| Q_{k}^{3}=q_{0}\right\}$, produce $t s u(3)$. The ternary hypercomplex units $|z \cdot \tilde{z} \cdot \tilde{z}|=1$ produce the ternary non-Abelian $\operatorname{TS} U(3)$ group Lee, respectively. The corresponding $T S U(3)$-invariant hypersurface $|z \cdot \tilde{z} \cdot \tilde{z}|=1$ takes the following form $[4,8]$ :

$$
F\left(x_{0}, \ldots, x_{8}\right)=\left|z_{0}\right|^{3}+\left|z_{1}\right|^{3}+\left|z_{2}\right|^{3}-\left(z_{0} \tilde{z}_{1} \tilde{z}_{2}\right)-\left(\tilde{z}_{0} \tilde{z}_{1} z_{2}\right)-\left(\tilde{z}_{0} \tilde{z}_{1} z_{2}\right)=1,
$$

where

$$
\begin{aligned}
& z_{0}=x_{0} q_{0}+x_{7} q+x_{8} q^{2}, \quad z_{1}=x_{1} q_{0}+x_{2} q+x_{3} q^{2}, \quad z_{2}=x_{4} q_{0}+x_{5} q+x_{6} q^{2} \\
& \begin{aligned}
F\left(x_{0}, \ldots, x_{8}\right) & =x_{0}^{3}+x_{7}^{3}+x_{8}^{3}+x_{1}^{3}+x_{2}^{3}+x_{3}^{3}+x_{4}^{3}+x_{5}^{3}+x_{6}^{3}-3 x_{0} x_{7} x_{8}-3 x_{1} x_{2} x_{3} \\
& -3 x_{0} x_{7} x_{8}-3 x_{0}\left(x_{1} x_{4}+x_{2} x_{5}+x_{3} x_{6}\right) \\
& -3 x_{7}\left(x_{1} x_{5}+x_{2} x_{6}+x_{3} x_{4}\right)-3 x_{8}\left(x_{1} x_{6}+x_{2} x_{4}+x_{3} x_{5}\right)=1
\end{aligned}
\end{aligned}
$$

The result of correctness was the vanishing of all contributions $V_{k}$ in the decomposition:

$$
V=F\left(x_{0}, x_{1}, \ldots, x_{8}\right) Q_{0}+V_{1}\left(x_{0}, x_{1}, \ldots, x_{8}\right) Q_{1}+\ldots+V_{8}\left(x_{0}, x_{1}, \ldots, x_{8}\right) Q_{8},
$$

that is, out of the possible 729 terms, only 45 terms have remained non-zero! Thus, the constructed generators $Q_{a} ; a=1, \ldots$, have more complicated than binary quaternions, the commutation relations $Q_{a} Q_{b}=j^{k} Q_{b} Q_{a} ; j=e^{2 \pi i / 3}$, where the value of $k=0,1,2$ depends on the choice of the generator subgroup (there are three $\left\{Q_{1}, Q_{2}, Q_{3}\right\},\left\{Q_{4}, Q_{5}, Q_{6}\right\},\left\{Q_{7}, Q_{8}\right\}$, and they form the algebra tsu (3). The hypersurface itself is a group manifold defined by the ternary group $T S U(3)$. This group and its algebra are fundamentally different from the Cartan-Lie group of $S U(3)$ and its algebra $s u(3)$ defined by the 8 Gell-Mann generators, hand drawing). The corresponding cubic hypersurface takes the following form in $\mathbb{R}^{8}$ [8]:

$$
U=\left(\begin{array}{ccc}
z_{0} & q z_{1} & q^{2} z_{2} \\
q^{2} \tilde{z}_{2} & \tilde{z}_{0} & q \tilde{z}_{2} \\
q \tilde{\tilde{z}}_{1} & q^{2} \tilde{\tilde{z}}_{2} & \tilde{\tilde{z}}_{0}
\end{array}\right), \quad \operatorname{Det} U=F\left(x_{0}, \ldots, x_{8}\right) .
$$

Understanding of the existence of a new vacuum of the Abelian symmetries of the invisible light could help to find a generalization of the Lorentz group, that means, to go beyond the usual geometry of the 4-dimensional space-time. The conclusion in modern physics is that our quark-lepton matter forms our visible Universe, that is only a small part of a huge hyper-Universe [6,11]. Critical issues such as spin, charge, color, mass lead us to search for the new symmetric geometric spaces, the basics of which we are looking for in the searching for the new number theories, algebras, finite group symmetries and etc. If we are successful on this way, we can understand the mechanism of formation of our visible Universe?! Similarly, considering $n=4,5,6, \ldots$ ary hyper-complex numbers, one can construct $n$-ary algebras and n-ary groups with the corresponding commutation relations, where $j=e^{(2 \pi i / n)}$. The important property of the ternary (n-ary) group $T S U(3)$ in the matrix formalism is to introduce new concepts of Complex conjugation, Transposition, Hermitian conjugation and Unitarity: $U U^{+} U^{++}=1$. The next step was connected with the further study of ternary 
Clifford algebras in their relationship with binary Clifford algebras, which would allow one to build n-spinors material matter with unusual quantum properties. As an illustration, the first example of how the interpretation of the existing 3 neutrino states[10][11] may change assuming that the space-time properties of 3 neutrinos are different from the properties of charged quarks and charged leptons. So we can consider three neutrinos as a single field in a space of dimension $\mathrm{D}=6$ (12), i.e. with two, three additional non-compact measurements, and, in accordance with ternary complexity, the implementation (realization) of the neutrino can be represented as a 3-spinor with the following three "charge conjugation" operations (new neutrino light):

$$
\psi=\left(\begin{array}{c}
v_{e} \\
\tilde{v}_{\mu} \\
\tilde{\tilde{v}}_{\tau}
\end{array}\right), \psi^{C}=\left(\begin{array}{c}
v_{\tau} \\
\tilde{v}_{e} \\
\tilde{\tilde{v}}_{\mu}
\end{array}\right), \psi^{C C}=\left(\begin{array}{c}
v_{\mu} \\
\tilde{v}_{\tau} \\
\tilde{\tilde{v}}_{e}
\end{array}\right), \psi^{C C C}=\psi
$$

describing a system of three neutrino states $v_{e}$-neutrino, $v_{\mu}$ - neutrino, $v_{\tau}$ neutrino, which can be represented as a particle-antiparticle-anti-particle (ternary model of 3 neutrino states, by analogy with the 4-dimensional Dirac theory of the electron. The representation of three neutrino states in the $\mathrm{D}=6$ assumes the existence of a new Abelian group $U(1)$ associated with the existence of a new light, possibly associated with dark energy.

\section{References}

[1] G. Volkov, Int.J. Mod. Phys A 19, 4835-4860 (2004). hep-th/0402042

[2] A. Dubrovskiy and G. Volkov, Adv.Appl.Clifford Algebras 17, 159-181 (2007)

[3] L.N. Lipatov, M. Rausch de Traunbenberg, G. Volkov, J. Math. Phys. 49, 013502 (2008)

[4] G. Volkov, On the complexifications of the Euclidean $R^{n}$ spaces and the $n$-dimensional generalization of Pythagore theorem, arXiv:1006.5630 [math-ph] (2010)

[5] V. Samoylenko and G.Volkov, The GUT of the light: On the Abelian Complexifications of the Euclidean $R^{n}$ spaces, arXiv:0912.2037 [physics.gen-ph]

[6] S.V. Smurov, G.G. Volkov, I.O. Glotova, S.N. Kukin, A.R. Muradova, Izvestiya IIP (Serpukhov) 4(38), 71-84 (2015)

[7] G.G. Volkov, I.O. Glotova, S.N. Kukin, A.R. Muradova, Izvestiya IIP (Serpukhov) 2(40), 75-84 (2016)

[8] G. Volkov, Ternary "Quaternions" and Ternary TU(3) algebra, arXiv:1006.5627 [math-ph]

[9] A.A. Maslikov and G.G. Volkov, A.A. Maslikov, G.G. Volkov, Nonlinear Dynamics and Applications 20, 257-264 (2014)

[10] G. Volkov, The possible signals from $D=6$, arXiv:1112.3583 [hep-ph]

[11] K. Dadochkina, S.V. Smurov, G. Volkov, in Proc. of MINSK NPCS-XXV, 2018 